\title{
Interference Cancellation Using Replica Signal for HTRCI-MIMO/OFDM in Time-Variant Large Delay Spread Longer Than Guard Interval
}

\author{
Yuta Ida, ${ }^{1}$ Chang-Jun Ahn, ${ }^{2}$ Takeshi Kamio, ${ }^{1}$ Hisato Fujisaka, ${ }^{1}$ and Kazuhisa Haeiwa ${ }^{1}$ \\ ${ }^{1}$ Graduate School of Information Sciences, Hiroshima City University, 3-4-1 Ozukahigashi, Asaminami-ku, \\ Hiroshima 731-3194, Japan \\ ${ }^{2}$ Graduate School of Engineering, Chiba University, 1-33 Yayoi-cho, Inage-ku, Chiba 263-8522, Japan
}

Correspondence should be addressed to Yuta Ida, y.ida@chiba-u.jp

Received 15 July 2011; Revised 22 February 2012; Accepted 12 March 2012

Academic Editor: Hanho Lee

Copyright () 2012 Yuta Ida et al. This is an open access article distributed under the Creative Commons Attribution License, which permits unrestricted use, distribution, and reproduction in any medium, provided the original work is properly cited.

\begin{abstract}
Orthogonal frequency division multiplexing (OFDM) and multiple-input multiple-output (MIMO) are generally known as the effective techniques for high data rate services. In MIMO/OFDM systems, the channel estimation (CE) is very important to obtain an accurate channel state information (CSI). However, since the orthogonal pilot-based CE requires the large number of pilot symbols, the total transmission rate is degraded. To mitigate this problem, a high time resolution carrier interferometry (HTRCI) for MIMO/OFDM has been proposed. In wireless communication systems, if the maximum delay spread is longer than the guard interval (GI), the system performance is significantly degraded due to the intersymbol interference (ISI) and intercarrier interference (ICI). However, the conventional HTRCI-MIMO/OFDM does not consider the case with the time-variant large delay spread longer than the GI. In this paper, we propose the ISI and ICI compensation methods for a HTRCI-MIMO/OFDM in the time-variant large delay spread longer than the GI.
\end{abstract}

\section{Introduction}

Recently, the sophisticated terminal as a smart phone becomes widely used and many multimedia services are provided $[1,2]$. High speed packet access (HSPA) using a wideband code division multiplexing access (W-CDMA) is used in the mobile communications and provides the data services with the maximum transmission rate of about $14 \mathrm{Mbps}$ [3]. However, since the available frequency band is limited, a HSPA cannot improve the transmission rate more than now [4]. To solve this problem, orthogonal frequency division multiplexing (OFDM) and multiple-input multiple-output (MIMO) are actively used [5-9]. OFDM is a multicarrier digital modulation. The OFDM signal can be transmitted in parallel by using the many subcarriers that are mutually orthogonal. In MIMO systems, the signal of the several transmit antennas is transmitted in the same frequency band. Moreover, since each transmitted signal is sent over the independent channel, the space diversity can be obtained in the receiver. Therefore, a long term evolution (LTE) has been standardized as $3.9 \mathrm{G}$ system using MIMO/OFDM systems $[10,11]$. An LTE provides the maximum transmission rate of about $50-100$ Mbps. Moreover, an LTE Advanced will support broadband data services with the maximum transmission rate of about $100 \mathrm{M}-1$ Gbps as $4 \mathrm{G}$ systems.

Since the received signal is changed due to the amplitude and phase variations for a frequency selective fading, the channel estimation (CE) is important to compensate the channel variance. In the conventional MIMO/OFDM, the orthogonal pilot symbols-based CE is used to identify an accurate channel state information (CSI) [7]. However, since the orthogonal pilot-based CE requires the large number of pilot symbols and large transmission power, the transmission rate is degraded. To mitigate these problems, a high time resolution carrier interferometry (HTRCI) for MIMO/OFDM has been proposed [12]. 


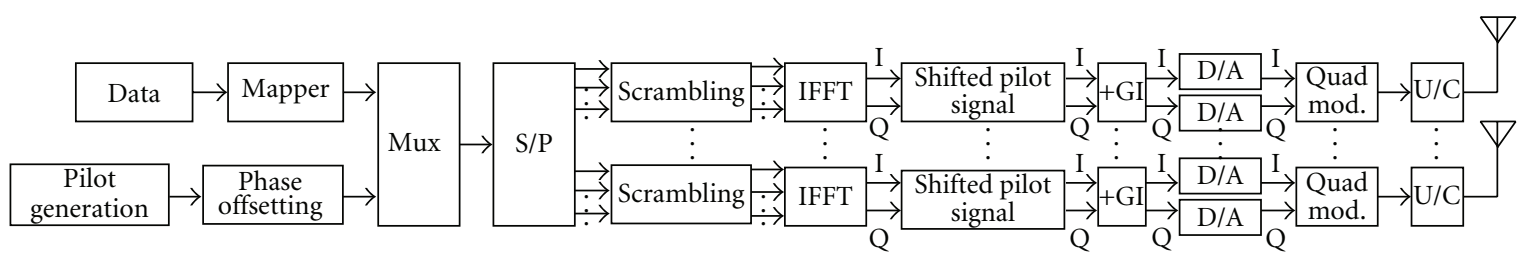

(a) Transmitter

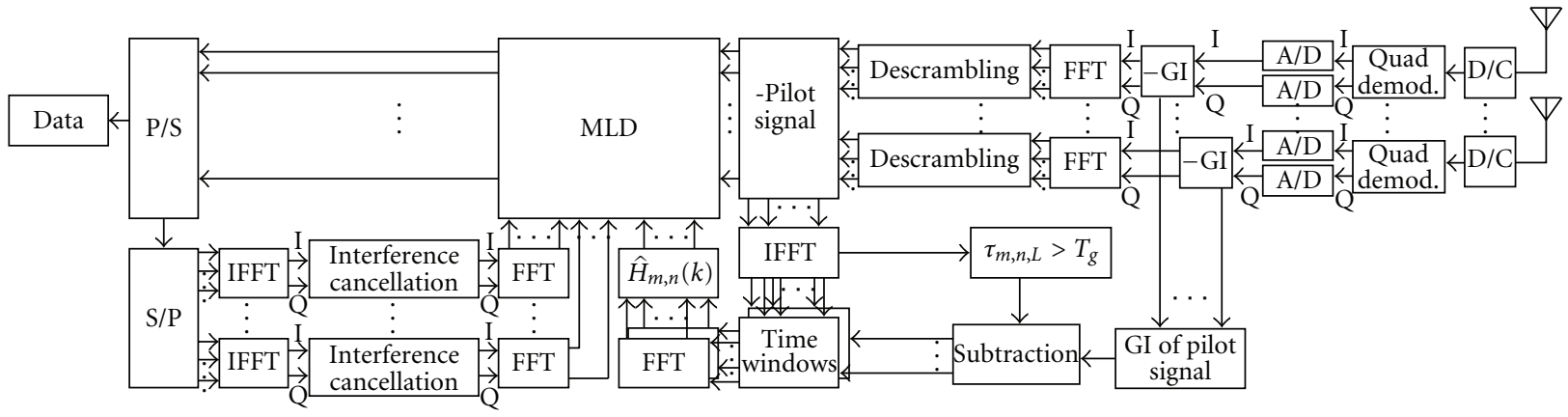

(b) Receiver

Figure 1: Proposed system.

In wireless communications, intersymbol interference (ISI) and intercarrier interference (ICI) are serious problems to mitigate the system performance. To prevent these problems, a guard interval (GI) is generally inserted. In general, GI is usually designed to be longer than the delay spread of the channel. However, if the maximum delay spread is longer than the GI, the system performance is significantly degraded due to the ISI and ICI. However, the conventional HTRCI-MIMO/OFDM does not consider the case with the time-variant large delay spread longer than the GI. In this paper, we propose the ISI and ICI compensation methods for a HTRCI-MIMO/OFDM in the time-variant large delay spread longer than the GI. Until this time, several schemes have proposed the ISI and ICI compensation methods due to the large delay spread channel. For example, [13] has proposed the ISI reduction method by extending the GI. However, since [13] has extended the GI length, the maximum throughput is degraded and the transmission power is also increased. Reference [14] has proposed the ISI and ICI compensation methods using the turbo equalization. However, [14] has large complexity by the iterative processing for the turbo equalizer. Reference [15] has proposed the ISI and ICI compensation methods using the estimated channel coefficients and the CSI to reproduce the interference components. However, the packet length becomes longer by using the training symbol. To mitigate the above-mentioned problems, we propose the time domain ISI compensation method with the replica signal based on the ICI compensation for a HTRCI-MIMO/OFDM in this paper. This paper is organized as follows. In Section 2, we present the system model. Then, we describe the proposed system in Section 3. In Section 4, we show the computer simulation results. Finally, the conclusion is given in Section 5 .

\section{System Model}

This section describes the system model, which employs the time-division multiplexing (TDM) transmission for multiple users. This system is illustrated in Figure 1.

2.1. Channel Model. We assume that a propagation channel consists of $L$ discrete paths with different time delays. The impulse response between the $m$ th transmit and $n$th receive antenna $h_{m, n}(\tau, t)$ is represented as follows:

$$
h_{m, n}(\tau, t)=\sum_{l=0}^{L-1} h_{m, n, l}(t) \delta\left(\tau-\tau_{m, n, l}\right),
$$

where $h_{m, n, l}, \tau_{m, n, l}$ are the complex channel gain and the time delay of the $l$ th propagation path, and $\sum_{l=0}^{L-1} E\left|h_{m, n, l}^{2}\right|=1$, where $E|\cdot|$ denotes the ensemble average operation. The channel transfer function $H_{m, n}(f, t)$ is the Fourier transform of $h_{m, n}(\tau, t)$ and is given by

$$
\begin{aligned}
H_{m, n}(f, t) & =\int_{0}^{\infty} h_{m, n}(\tau, t) \exp (-j 2 \pi f \tau) d \tau \\
& =\sum_{l=0}^{L-1} h_{m, n, l}(t) \exp \left(-j 2 \pi f \tau_{m, n, l}\right) .
\end{aligned}
$$

2.2. HTRCI-MIMO/OFDM. The transmission block diagram of the proposed system is shown in Figure 1(a). Firstly, the coded binary information data sequence is modulated, and $N_{p}$ pilot symbols are appended at the beginning of the sequence. The HTRCI-MIMO/OFDM transmitted signal for 
the $m$ th transmit antenna can be expressed in its equivalent baseband representation as follows:

$$
\begin{aligned}
s_{m}(t)=\sum_{i=0}^{N_{p}+N_{d}-1} g(t-i T) \cdot\left\{\sqrt{\frac{2 S}{N_{c}}} \sum_{k=0}^{N_{c}-1} u_{m}(k, i)\right. \\
\left.\cdot \exp \left[\frac{j 2 \pi(t-i T) k}{T_{s}}\right]\right\},
\end{aligned}
$$

where $N_{d}$ and $N_{p}$ are the number of data and pilot symbols, $N_{c}$ is the number of subcarriers, $T_{s}$ is the effective symbol length, $S$ is the average transmission power, and $T$ is the OFDM symbol length, respectively. The frequency separation between adjacent orthogonal subcarriers is $1 / T_{s}$ and can be expressed by using the $k$ th subcarrier of the $i$ th modulation symbol $d_{m}(k, i)$ with $\left|d_{m}(k, i)\right|=1$ for $N_{p} \leq i \leq$ $N_{p}+N_{d}-1$ as follows:

$$
u_{m}(k, i)=c_{\mathrm{PN}}(k) \cdot d_{m}(k, i),
$$

where $c_{\mathrm{PN}}$ is a long pseudonoise (PN) sequence as a scrambling code to reduce the peak-to-average power ratio (PAPR). GI is inserted in order to eliminate the ISI due to a multipath fading, and hence, we have

$$
T=T_{s}+T_{g},
$$

where $T_{g}$ is the GI length. In communication systems, $\varepsilon$ is generally considered as 4 or 5 , where $\varepsilon=T_{s} / T_{g}$. In this paper, we assume $\varepsilon=4$. In (3), the transmission pulse $g(t)$ is given by

$$
g(t)= \begin{cases}1 & \text { for }-T_{g} \leq t \leq T_{s} \\ 0 & \text { otherwise }\end{cases}
$$

For $0 \leq i \leq N_{p}-1$, the transmitted pilot signal of the $k$ th subcarrier for the $m$ th transmit antenna element is given by

$$
d_{m}(k, i)= \begin{cases}\sum_{\mu=0}^{\zeta-1} \exp \left(\frac{-j 2 \pi(\tilde{m}+2 \mu) T_{g} k}{T_{s}}\right) & \text { for } i=\left\lfloor\frac{m}{\zeta}\right\rfloor \\ 0 & \text { otherwise, }\end{cases}
$$

where $\zeta=\lfloor\varepsilon / 2\rfloor, \tilde{m}=\bmod (m, \zeta)$, and $\lfloor x\rfloor$ stands for the integer lower and closer to $x$, respectively. In this case, the HTRCI-MIMO/OFDM pilot signal can multiplex the same impulse responses from the transmit antenna elements in each receive antenna element in $\zeta$ times on the time domain without overlapping to each other. For example, if we consider $\varepsilon=4$ and 2 transmit antenna elements, we obtain $\sum_{k=0}^{N_{c}-1} d_{0}(k, 0)=\{1,0, \ldots, 1,0\}$ and $\sum_{k=0}^{N_{c}-1} d_{1}(k, 0)=$ $\{1,0,-1,0, \ldots, 1,0,-1,0\}$ as the pilot signal of the first and second transmit antenna elements from (7) as shown in Figure 2(a). In this case, since each pilot signal contains " 0 " components, we can identify the half of transmission power compared with the conventional MIMO/OFDM system using the orthogonal pilot. However, if $\tau_{m, n, L}>T_{g}$, the conventional HTRCI-MIMO/OFDM can not obtain an accurate CSI, where $\tau_{m, n, L}$ is the maximum delay spread for the $m$ th transmit and $n$th receive antenna. To solve this problem, we propose the new channel estimation using a HTRCI-MIMO/OFDM. Firstly, in the transmitter, we shift the channel impulse responses of (7) as follows:

$$
d_{m}(k, i)= \begin{cases}\sum_{\mu=0}^{\zeta-1} \exp \left(\frac{-j 2 \pi(2 \tilde{m}+\mu) T_{g} k}{T_{s}}\right) & \text { for } i=\left\lfloor\frac{m}{\zeta}\right\rfloor \\ 0 & \text { otherwise. }\end{cases}
$$

In (8), the channel impulse responses are shifted as shown in Figure 2(a). This operation enables estimating the maximum delay spread longer than the GI and to obtain an accurate CSI in the receiver.

The received structure is illustrated in Figure 1(b). By applying the FFT operation, the received signal $r_{n}(t)$ is resolved into $N_{c}$ subcarriers. The received signal for the $n$th receive antenna $r_{n}(t)$ in the equivalent baseband representation can be expressed as follows:

$$
r_{n}(t)=\sum_{m=0}^{M-1} \int_{-\infty}^{\infty} h_{m, n}(\tau, t) s_{m}(t-\tau) d \tau+n_{n}(t),
$$

where $M$ is the number of transmit antennas and $n_{n}(t)$ is additive white Gaussian noise (AWGN) with a single-sided power spectral density of $N_{0}$ for the $n$th receive antenna, respectively. The $k$ th subcarrier $\widetilde{r}_{n}(k, i)$ is given by

$$
\begin{aligned}
\tilde{r}_{n}(k, i)= & \frac{1}{T_{s}} \int_{i T}^{i T+T_{s}} r_{n}(t) \exp \left[\frac{-j 2 \pi(t-i T) k}{T_{s}}\right] d t \\
= & \sqrt{\frac{2 S}{N_{c}}} \sum_{m=0}^{M-1} \sum_{e=0}^{N_{c}-1} u_{m}(e, i) \\
& \cdot \frac{1}{T_{s}} \int_{0}^{T_{s}} \exp \left[j 2 \pi \cdot \frac{(e-k) t}{T_{s}}\right] \\
& \cdot\left\{\int_{-\infty}^{\infty} h_{m, n}(\tau, t+i T) g(t-\tau)\right. \\
& \left.\cdot \exp \left(\frac{-j 2 \pi e \tau}{T_{s}}\right) d \tau\right\} d t+\hat{n}_{n}(k, i),
\end{aligned}
$$

where $\hat{n}_{n}(k, i)$ is AWGN noise with zero mean and a variance of $2 N_{0} / T_{s}$. After abbreviating, (10) can be rewritten as follows:

$$
\begin{aligned}
& \tilde{r}_{n}(k, i) \approx \frac{1}{T_{s}} \sqrt{\frac{2 S}{N_{c}}} \sum_{m=0}^{M-1} \sum_{e=0}^{N_{c}-1} u_{m}(e, i) \\
& \cdot \int_{0}^{T_{s}} \exp \left[j 2 \pi \cdot \frac{(e-k) t}{T_{s}}\right] \\
& \cdot\left\{\int_{-\infty}^{\infty} h_{m, n}(\tau, t+i T) g(t-\tau)\right. \\
&\left.\cdot \exp \left(\frac{-j 2 \pi e \tau}{T_{s}}\right) d \tau\right\} d t+\hat{n}_{n}(k, i) \\
&=\sqrt{\frac{2 S}{N_{c}}} \sum_{m=0}^{M-1} H_{m, n}\left(\frac{k}{T_{s}}, i T\right) u_{m}(k, i)+\hat{n}_{n}(k, i) .
\end{aligned}
$$




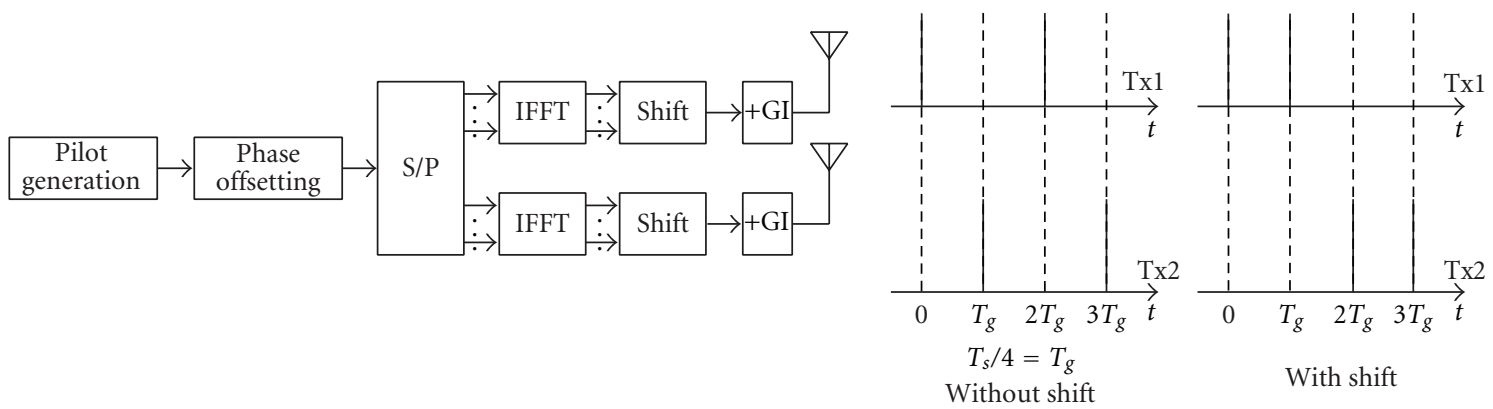

(a) Transmitter
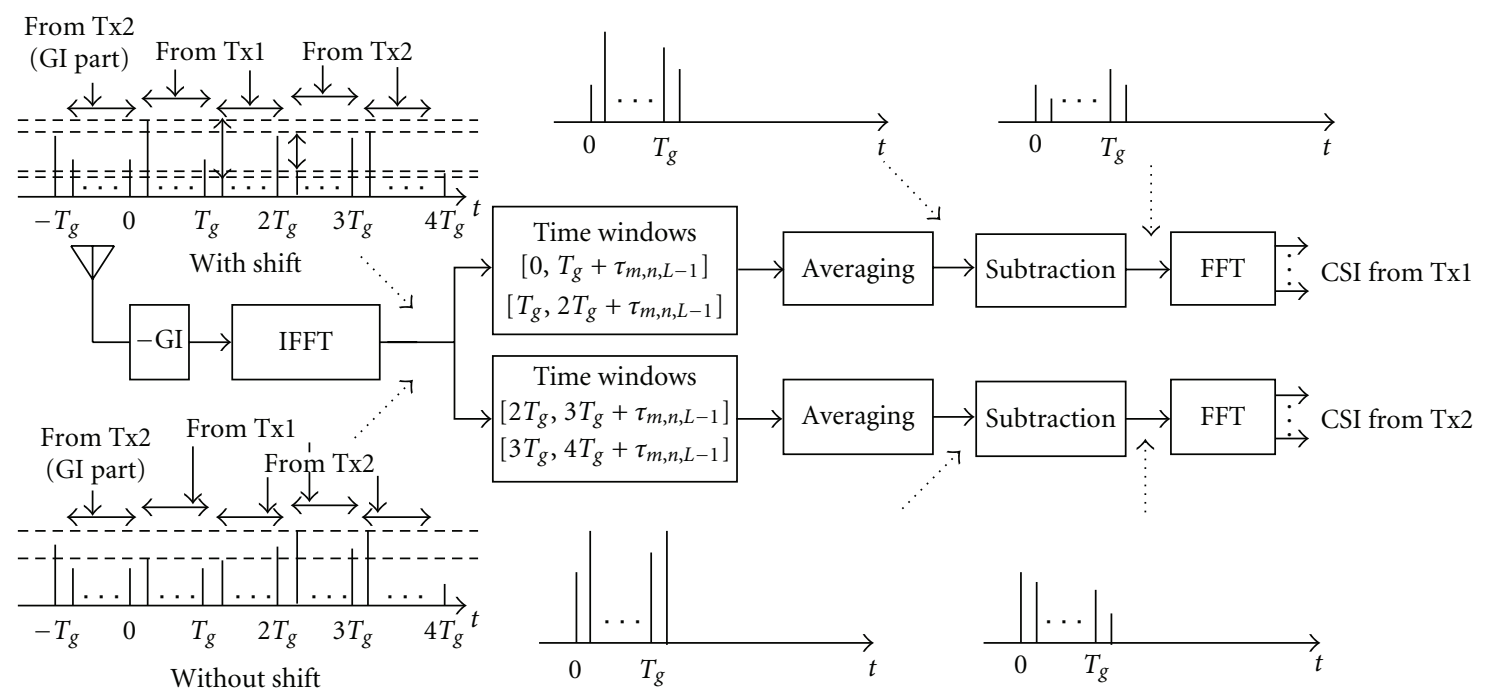

(b) Receiver

Figure 2: The concept of a HTRCI-MIMO/OFDM to apply the maximum delay spread longer than the GI for 2 transmit antenna elements and $\varepsilon=4$.

After descrambling, the output signal $\hat{r}_{n}(k, i)$ for the $n$th receive antenna element is given by

$$
\begin{aligned}
\hat{r}_{n}(k, i) & =\frac{c_{\mathrm{PN}}^{*}(k)}{\left|c_{\mathrm{PN}}(k)\right|^{2}} \tilde{r}_{n}(k, i) \\
& =\sqrt{\frac{2 S}{N_{c}}} \sum_{m=0}^{M-1} H_{m, n}\left(\frac{k}{T_{s}}, i T\right) d_{m}(k, i)+\hat{n}_{n}(k, i),
\end{aligned}
$$

where $(\cdot)^{*}$ is a complex conjugate and $c_{\mathrm{PN}}^{*}(k) /\left|c_{\mathrm{PN}}(k)\right|^{2}$ is the descrambling operation, respectively. Observing (11) and (12), the noise components are the same notation. In this paper, the descrambling operation is to rotate the phase of each subcarrier by using a PN code. Since $\varepsilon=T_{s} / T_{g}$, a HTRCI-MIMO/OFDM can multiplex the same impulse responses in $\zeta$ times on the time domain. After the pilot signal separation, the pilot signal is converted to the time domain signal $\hat{r}_{n}(t)$ again as

$$
\begin{aligned}
\hat{r}_{n}(t)= & \sum_{i=0}^{N_{p}-1} \sqrt{\frac{2 P}{N_{c}}} \sum_{k=0}^{N_{c}-1} \hat{r}_{n}(k, i) \exp \left[\frac{j 2 \pi(t-i T) k}{T_{s}}\right] \\
= & \sum_{i=0}^{N_{p}-1} \sqrt{\frac{2 P}{N_{c}}} \sum_{m=0}^{M-1} h_{m, n}(\tau, t+i T) \sum_{k=0}^{N_{c}-1} d_{m}(k, i) \\
& \cdot \exp \left[\frac{j 2 \pi(t-i T) k}{T_{s}}\right]+\tilde{n}_{n}(t) \\
= & \sum_{i=0}^{N_{p}-1} \sqrt{\frac{2 P}{N_{c}}} \sum_{m=0}^{M-1} \sum_{l=0}^{L-1} h_{m, n, l}(t+i T) \\
& \cdot \frac{1}{\sqrt{\zeta}}\left\{\sum_{\mu=0}^{\zeta-1} \delta\left(\tau-\tau_{m, n, l}-\tau_{(2 \tilde{m}+\mu) T_{g}}\right)\right\}+\tilde{n}_{n}(t),
\end{aligned}
$$

where $P$ is the transmission pilot signal power and $\tilde{n}_{n}(t)$ is the noise component, respectively. Here, if $\tau_{m, n, L}>T_{g}$, since 
the channel impulse responses of the different time window overlap the desired channel impulse responses, the accurate CSI can not be obtained. Therefore, we process as follows. At the receiver, in Figure 2(b), the overlapped channel impulse responses show the same amplitude for the transmitted pilot signal without the shift in the different time window. In this case, we can not estimate the maximum delay spread. On the other hand, the overlapped channel impulse responses show the different amplitude for the transmitted pilot signal with the shift in the different time window. Therefore, we can estimate the maximum delay spread from the different amplitude in the different time windows. Next, we eliminate the channel impulse responses of the different time window. The channel impulse responses of the different time window can be eliminated by using the channel impulse responses of the GI. These channel impulse responses do not contain the channel impulse responses of the different time window. This is because the pilot signal of the GI is the head of packet and it does not contain the channel impulse responses of the different time window. Therefore, we can eliminate the channel impulse responses of the different time window. From the above process, the frequency response of the $k$ th subcarrier between the $m$ th transmit and $n$th receive antenna $\hat{H}_{m, n}(k)$ for $\tau_{m, n, L}>T_{g}$ is obtained by

$$
\begin{aligned}
& \hat{H}_{m, n}(k) \\
& =\sqrt{\frac{N_{c} \zeta}{2 P} \sum_{m=0}^{M-1} \sum_{e=0}^{N_{c}-1} \frac{1}{T_{s}}} \\
& \cdot \int_{0}^{T_{s}}\left\{\sum_{l=0}^{L-1} \sum_{l^{\prime}=L^{\prime}-1}^{L-1} h_{m, n, l}(t+\rho T)\right. \\
& \cdot \int_{-\infty}^{\infty}\left\{\sum_{\mu=0}^{\zeta-1} \delta\left(\tau-\tau_{m, n, l}-\tau_{(2 \tilde{m}+\mu) T_{g}}\right)\right. \\
& \left.\left.\quad-\delta\left(\tau-\tau_{m, n, l^{\prime}}\right)\right\} \cdot \exp \left(\frac{-j 2 \pi e \tau}{T_{s}}\right) d \tau\right\} d t \\
& +\eta_{m, n}(k) \quad \text { for } L^{\prime} \leq T_{g}, \rho=\left\lfloor\frac{m}{\zeta}\right\rfloor
\end{aligned}
$$

where $\eta_{m, n}(k)$ is AWGN component with $E\left[\left|\eta_{m, n}(k)\right|\right]^{2}=$ $E\left[\left|\hat{n}_{n}(k, i) / \zeta\right|\right]^{2}=\sigma^{2} / \zeta$.

\section{Proposed System}

3.1. Rewritten Matrix Form. After the pilot signal separation, (13) for $\tau_{m, n, L} \leq T_{g}$ can be rewritten in the matrix form as follows:

$$
\mathbf{R}_{i, n}=\sum_{m=0}^{M-1} \lambda_{i, m, n} F \mathbf{D}_{i, m}+\mathbf{N}_{i, n}
$$

where $\lambda_{i, m, n}$ is the $N_{c} \times N_{c}$ time-domain channel matrix for the $i$ th symbol between the $m$ th transmit and $n$th receive antenna, $\mathbf{N}_{i, n}$ is the $N_{c} \times 1$ noise matrix, and $F$ is the IFFT operation, respectively. However, (15) for $\tau_{m, n, L}>T_{g}$ is rewritten as follows:

$$
\mathbf{R}_{i, n}=\sum_{m=0}^{M-1}\left(\lambda_{\mathrm{isi}, i-1, m, n} F \mathbf{D}_{i-1, m}+\lambda_{\mathrm{ici}, i, m, n} F \mathbf{D}_{i, m}\right)+\mathbf{N}_{i, n},
$$

where $\boldsymbol{\lambda}_{\mathrm{isi}, i-1, m, n}, \boldsymbol{\lambda}_{\mathrm{ici}, i, m, n}$ denote the ISI and ICI channel matrices for the $(i-1)$ th and $i$ th symbols between the $m$ th transmit and $n$th receive antenna, respectively. Observing (16), since the received signal $\mathbf{R}_{i, n}$ contains the ISI and ICI terms, these equalization processing are necessary.

3.2. ISI and ICI Equalization. In $\tau_{m, n, L}>T_{g}$, since the first data symbol of $\mathbf{R}_{i, n}$ does not contain the ISI [16], (16) for $i=0$ is obtained as

$$
\mathbf{R}_{0, n}=\sum_{m=0}^{M-1} \lambda_{\mathrm{ici}, 0, m, n} F \mathbf{D}_{0, m}+\mathbf{N}_{0, n}
$$

However, the data symbols for $i>0$ have the ISI. Here, the ISI equalization is performed by using the previous detected symbol $\overline{\mathbf{D}}_{i-1, m, n}$ and the estimated ISI channel matrix $\hat{\lambda}_{\text {isi, } m, n}$. The estimated ISI channel matrix $\hat{\lambda}_{\text {isi, } m, n}$ consists of the estimated channel impulse response. The estimated channel impulse response $\hat{h}_{m, n, l^{\prime}}(t) \delta\left(\tau-\tau_{m, n, l^{\prime}}\right)$ is obtained by the channel response $\hat{H}_{m, n}(k)$ after the IFFT operation, where $L^{\prime} \leq l^{\prime} \leq L-1$. Therefore, the ISI equalized signal $\widetilde{\mathbf{R}}_{i, n}$ is given by

$$
\begin{aligned}
\widetilde{\mathbf{R}}_{i, n} & =\mathbf{R}_{i, n}-\sum_{m=0}^{M-1} \hat{\lambda}_{\mathrm{isi}, m, n} F \overline{\mathbf{D}}_{i-1, m} \\
& =\sum_{m=0}^{M-1} \lambda_{\mathrm{ici}, i, m, n} F \mathbf{D}_{i, m}+\tilde{\mathbf{N}}_{i, n},
\end{aligned}
$$

where $\tilde{\mathbf{N}}_{i, n}$ is the noise term with the residual ISI. After the FFT operation, by using maximum likelihood detection (MLD), the detected signal $\widetilde{\mathbf{D}}_{i}=\left[\widetilde{\mathbf{D}}_{i, 0}, \ldots, \widetilde{\mathbf{D}}_{i, m}, \ldots, \widetilde{\mathbf{D}}_{i, M-1}\right]^{T}$ is obtained as follows:

$$
\widetilde{\mathbf{D}}_{i}=\arg \min _{\hat{\mathbf{D}}} \sum_{n=0}^{N-1} \sum_{m=0}^{M-1}\left\|F^{-1} \widetilde{\mathbf{R}}_{i, n}-\hat{\mathbf{H}}_{m, n} \hat{\mathbf{D}}_{i, m}\right\|^{2}
$$

where $(\cdot)^{T}$ is the transpose operation, $N$ is the number of receive antennas, $\hat{\mathbf{D}}_{i, m}$ is the constellation of the symbol replica candidates as $\sum_{m=0}^{M-1} \sum_{c=0}^{C-1} \mathbf{D}_{i, m, c}, C$ is the modulation level, and $\hat{\mathbf{H}}_{m, n}$ is the matrix form of $\hat{H}_{m, n}(k)$, respectively. From (19), ISI is eliminated from the received signal. However, the orthogonality is destroyed by the detected signal due to the ISI compensation. If ICI is eliminated from (19), the detected signal $\widetilde{\mathbf{D}}_{i, m}$ can be more accurately detected.

3.3. Replica Signal Insertion Based on ICI Equalization. To eliminate the ICI, we consider the orthogonality reconstruction with inserting the detected signal after the ISI 
compensation. The ICI equalized signal $\breve{\mathbf{R}}_{i, n}$ with inserting eliminated the part of signal using the previous detected symbol $\widetilde{\mathbf{D}}_{i, m}$ is given by

$$
\begin{aligned}
\breve{\mathbf{R}}_{i, n} & =\widetilde{\mathbf{R}}_{i, n}+\sum_{m=0}^{M-1} \hat{\boldsymbol{\lambda}}_{\mathrm{ici}, m, n} F \widetilde{\mathbf{D}}_{i, m} \\
& =\sum_{m=0}^{M-1} \boldsymbol{\lambda}_{i, m, n} F \mathbf{D}_{i, m}+\breve{\mathbf{N}}_{i, n},
\end{aligned}
$$

where $\hat{\boldsymbol{\lambda}}_{\text {ici, }, m, n}$ is the estimated ICI channel matrix and $\breve{\mathbf{N}}_{i, n}$ is the noise term with the residual ISI and residual ICI, respectively. $\hat{\lambda}_{\text {ici, }, m, n}$ consists of the estimated channel impulse responses $\hat{h}_{m, n, l^{\prime}}(t) \delta\left(\tau-\tau_{m, n, l^{\prime}}\right)$. After the FFT operation, the detected signal $\breve{\mathbf{D}}_{i}=\left[\breve{\mathbf{D}}_{i, 0}, \ldots, \breve{\mathbf{D}}_{i, m}, \ldots, \breve{\mathbf{D}}_{i, M-1}\right]^{T}$ is obtained as follows:

$$
\breve{\mathbf{D}}_{i}=\arg \min _{\hat{\mathbf{D}}} \sum_{n=0}^{N-1} \sum_{m=0}^{M-1}\left\|F^{-1} \breve{\mathbf{R}}_{i, n}-\hat{\mathbf{H}}_{m, n} \hat{\mathbf{D}}_{i, m}\right\|^{2} .
$$

Observing (19) and (21), since ICI is eliminated, $\breve{\mathbf{D}}_{i, m}$ is the more accurately detected signal compared with $\widetilde{\mathbf{D}}_{i, m}$.

3.4. Complexity Comparison. Here, we compare the complexity of the conventional and proposed methods. Firstly, we show the complexity of [14]. The authors of [14] have proposed the ISI and ICI compensations using the turbo equalization. From Table 1 of [14], the complexity of [14] is given by

$$
\begin{aligned}
C_{[14]}= & \left\{N N_{s} M N_{c}+4 N_{c} N M^{2}+3 N_{c} N M\right. \\
& \left.+N N_{c} \log _{2} N_{c}+6 N\left(N_{s}+L\right) M N_{c}\right\} N_{d} \\
= & N N_{c} N_{d}\left\{M\left(7 N_{s}+4 M+6 L+3\right)+\log _{2} N_{c}\right\},
\end{aligned}
$$

where $N_{s}$ is the number of sampling points for GI and effective symbol. Next, we show the complexity of our proposed method. Firstly, the complexity of (18) is obtained by

$$
C_{\text {isi }}=N N_{c}\left(N_{d}-1\right) C^{M}+\left\{M N_{c}\left(N_{d}-1\right)\right\} \log N_{c} .
$$

For (23), the first term is the complexity of MLD and the second term is the complexity of IFFT. Next, the complexity of (20) is obtained by

$$
\begin{aligned}
C_{\mathrm{ici}}= & N N_{c} N_{d} C^{M}+M N N_{c} N_{d} \log N_{c} \\
= & \left\{N N_{c}\left(N_{d}-1\right)+N N_{c}\right\} C^{M} \\
& +\left[\left\{M N_{c}\left(N_{d}-1\right)\right\}+M N_{c}\right] \log N_{c} .
\end{aligned}
$$

For (24), the first and second terms are the complexity of MLD and IFFT of (23). Finally, the complexity of (21) is

$$
C_{\mathrm{MLD}}=N N_{c} N_{d} C^{M}=\left\{N N_{c}\left(N_{d}-1\right)+N N_{c}\right\} C^{M} .
$$

Therefore, the complexity of the proposed method is obtained by

$$
\begin{aligned}
C_{\text {pro }} & =C_{\text {isi }}+C_{\text {ici }}+C_{M L D} \\
& =N_{c}\left\{N\left(3 N_{d}-1\right) C^{M}+M\left(N_{d}-1\right) \log N_{c}\right\} .
\end{aligned}
$$

For example, when $M=N=2, N_{c}=64, N_{d}=20, N_{s}=$ $80, L=2$, and $C=4$, the complexities of [14] and the proposed method are 3000320 and 130560 from (22) and (26). Therefore, the proposed method is small compared with [14].

\section{Computer Simulated Results}

In this section, we show the performance of the proposed method. Figure 1 shows the simulation model of the proposed system. In this simulation, we assumed that $2 \times 2$ and $4 \times 4$ MIMO systems. On the transmitter, the pilot signal is assigned for each transmitter using (8). In this case, the proposed system can multiplex the same impulse responses from the transmit antenna elements in each receive antenna element in $\zeta$ times on the time domain. These have been found to be efficient for the transmission of the OFDM signal over the frequency selective fading channel. After serial to parallel (S/P) converted, the coded bits are QPSK modulated, and then the pilot signal and data signal are multiplexed with the scrambling using a PN code to reduce the PAPR. The OFDM time signal is generated by the IFFT operation and is transmitted to the frequency-selective and time-variant radio channel after the cyclic extension has been inserted. The transmitted signal is subject to the broadband channel propagation. In this simulation, we assume that OFDM symbol period is $5 \mu \mathrm{s}$ and guard interval is $1 \mu \mathrm{s}$ for $\varepsilon=4$, and $L=2,3$ path Rayleigh fadings. The maximum Doppler frequency is $10 \mathrm{~Hz}$. In the receiver, guard interval is erased from the received signal and the received signal is converted S/P. The parallel sequences are passed to the FFT operator and convert the signal back to the frequency domain. After the descrambling and IFFT operation, each impulse response for all combination of the transmit and receive antenna elements can be estimated by extracting and averages $\zeta$ times impulse responses using the time windows with (14). In $\tau_{m, n, L}>T_{g}$, the HTRCI-MIMO/OFDM pilot signal contains the overlapped channel impulse responses from the different time window. However, the pilot signal of the GI does not contain the overlapped channel impulse responses. By using this signal, the channel impulse responses of the different time window are eliminated from the overlapped channel impulse responses as shown in Figure 2(b). The frequency domain data signal is detected and demodulated by using the MLD algorithm. Since the detected data signal contains the ISI and ICI, these equalization processing are necessary. The ISI equalization is performed with the previous detected symbol and estimated ISI channel matrix as (18). From (18), ISI is eliminated. However, the orthogonality is destroyed by the detected signal due to the ISI compensation. To reconstruct the orthogonality, the ICI equalization is performed by using the replica signal insertion as (20). Finally, the data 


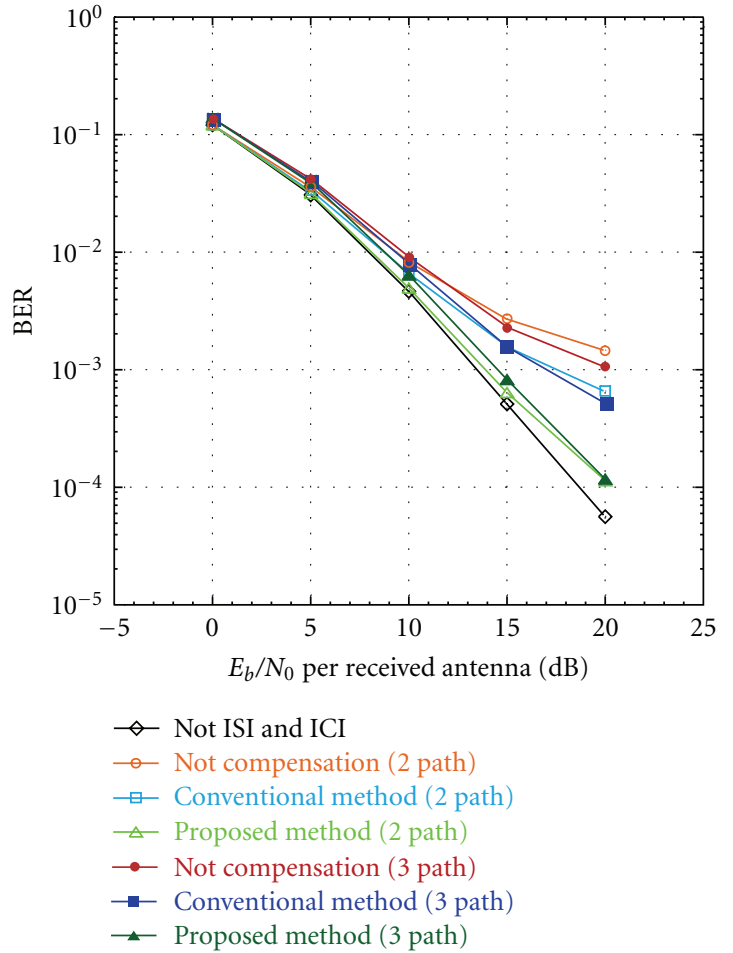

FIgURE 3: The BER of the conventional and proposed methods for $2 \times 2$ MIMO system at Doppler frequency of $10 \mathrm{~Hz}$.

TABLe 1: Simulation parameters.

\begin{tabular}{lc}
\hline Data modulation & QPSK \\
Data detection & MLD \\
Symbol duration & $5 \mu \mathrm{s}$ \\
Frame size & $N_{p}=1,2, N_{d}=20$ \\
FFT size & 64 \\
Number of carriers & 64 \\
Guard interval & 16 sample times \\
Fading & 2,3 path Rayleigh fading \\
Doppler frequency & $10 \mathrm{~Hz}$ \\
Antennas & $(M, N)=(2,2),(4,4)$ \\
\hline
\end{tabular}

signal is detected as (21). The packet consists of $N_{p}=1,2$ pilot symbols and $N_{d}=20$ data symbols. Table 1 shows the simulation parameters.

Figures 3 and 4 show the BER of the conventional and proposed methods for $2 \times 2$ and $4 \times 4$ MIMO systems at Doppler frequency of $10 \mathrm{~Hz}$. The number of maximum delay spread is 4 and 16 in $2 \times 2$ and $4 \times 4$ MIMO systems, respectively. From the simulation results, the BER performance for no ISI and ICI compensation is increased about 22 and 300 times compared with no ISI and ICI case in $2 \times 2$ and $4 \times 4$ MIMO systems, respectively. For the conventional method, the BER performance shows the error floor in high $E_{b} / N_{0}$. This is because the residual ISI and ICI are remained. The proposed method shows approximately the same BER performance compared with no ISI and ICI case. Therefore, the proposed method can eliminate the

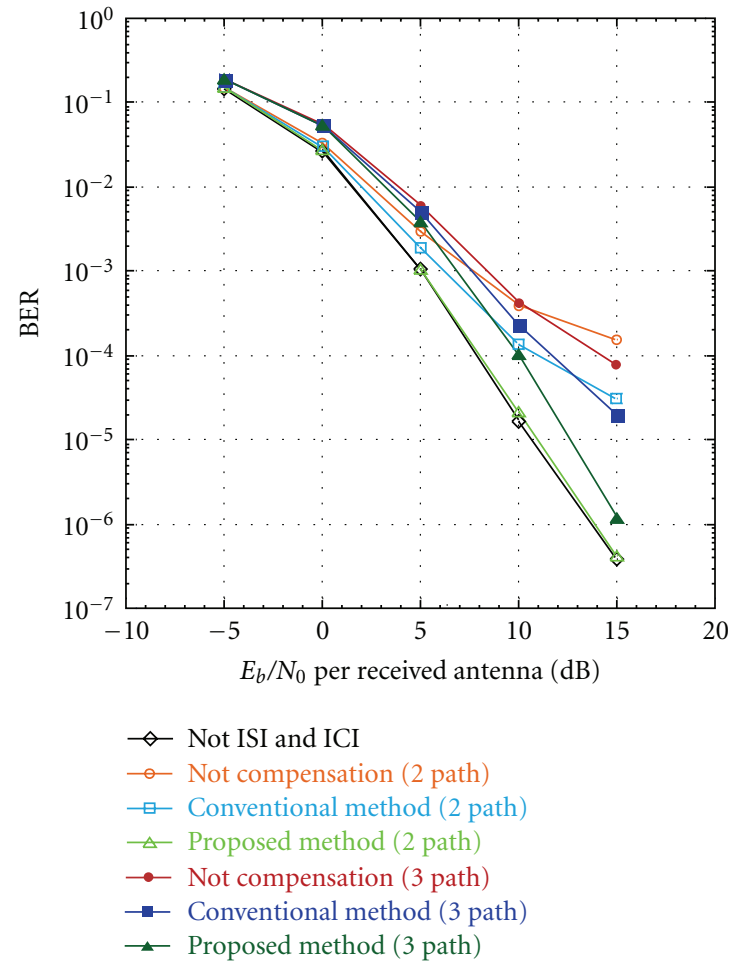

FIGURE 4: The BER of the conventional and proposed methods for $4 \times 4$ MIMO system at Doppler frequency of $10 \mathrm{~Hz}$.

ISI and ICI. Next, we compare the 2- and 3-path models. The 2-path model contains the channel impulse responses of the different time window in the time domain HTRCIMIMO/OFDM pilot signal. However, they do not overlap the desired channel impulse responses. On the other hand, the 3path model overlaps the desired channel impulse responses. Therefore, the channel impulse responses of the different time window are eliminated by using the channel impulse responses of the GI. From the simulation results, the 2-path model shows the better BER performance than that of the 3path model in low $E_{b} / N_{0}$. It means that CSI degrades due to the eliminated processing of the overlapped channel impulse responses in the 3-path model.

Figures 5 and 6 show the BER of the proposed method for $2 \times 2$ and $4 \times 4$ MIMO systems at Doppler frequency of $10 \mathrm{~Hz}$. The number of maximum delay spread is 4 and 16 in 2 $\times 2$ and $4 \times 4$ MIMO systems, respectively. For the proposed method without the noise, the noise is not added in the channel impulse responses of the GI. From the simulation results, the 2- and 3-path models without the noise show the approximately same BER performance. Therefore, the 3-path model with the noise degrades due to the noise of the GI.

Figures 7 and 8 show the BER versus the number of maximum delay spread for the conventional and proposed methods with $2 \times 2$ and $4 \times 4$ MIMO systems at Doppler frequency of $10 \mathrm{~Hz}$. Here, $E_{b} / N_{0}$ per received antenna is 20 and $15 \mathrm{dBs}$ in $2 \times 2$ and $4 \times 4 \mathrm{MIMO}$ systems, respectively. In $2 \times 2$ MIMO system, the BER performances of no ISI and ICI compensation and conventional method are increased 


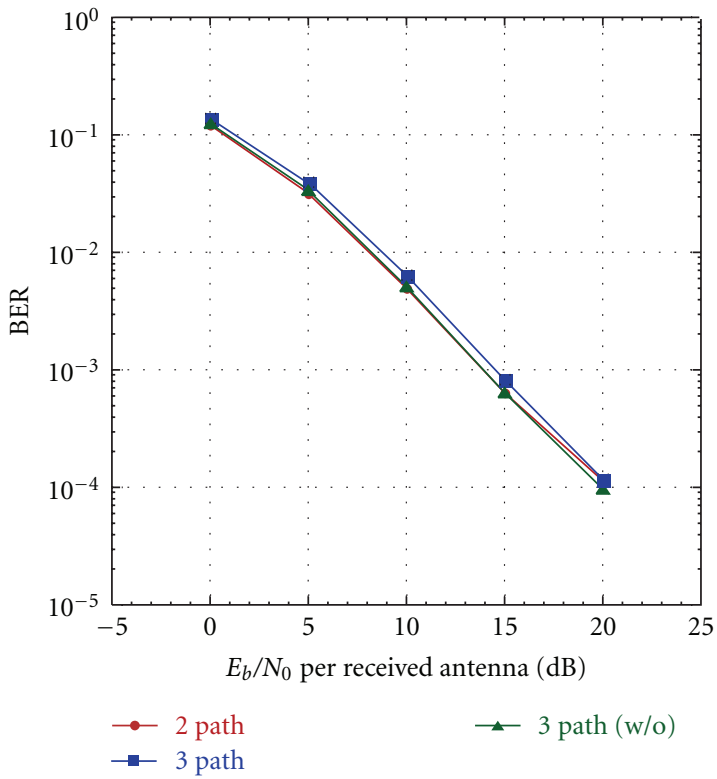

FIGURE 5: The BER of the proposed method for $2 \times 2$ MIMO system at Doppler frequency of $10 \mathrm{~Hz}$.

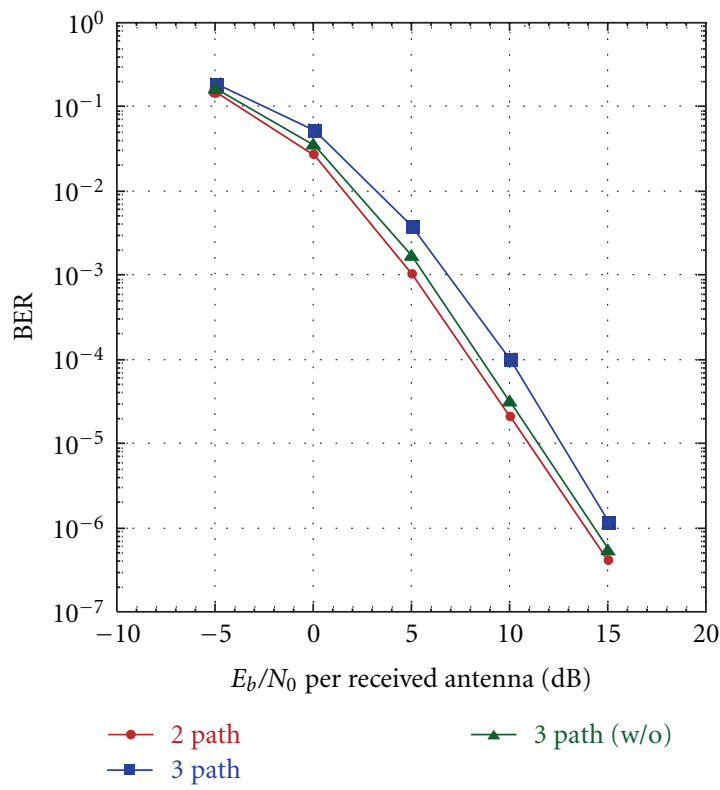

FIGURE 6: The BER of the proposed method for $4 \times 4$ MIMO system at Doppler frequency of $10 \mathrm{~Hz}$.

about 5 times. On the other hand, the BER performance of the proposed method is increased about 2 times. Therefore, the proposed method can suppress the ISI and ICI due to the changing of the number of maximum delay spread. In $4 \times 4 \mathrm{MIMO}$ system, the BER performance of no ISI and ICI compensation and the conventional method are increased about 170 and 80 times. On the other hand, the BER performance of the proposed method is increased about 4 times and the proposed method can suppress the ISI and ICI as $2 \times 2$ MIMO system. However, the BER performance

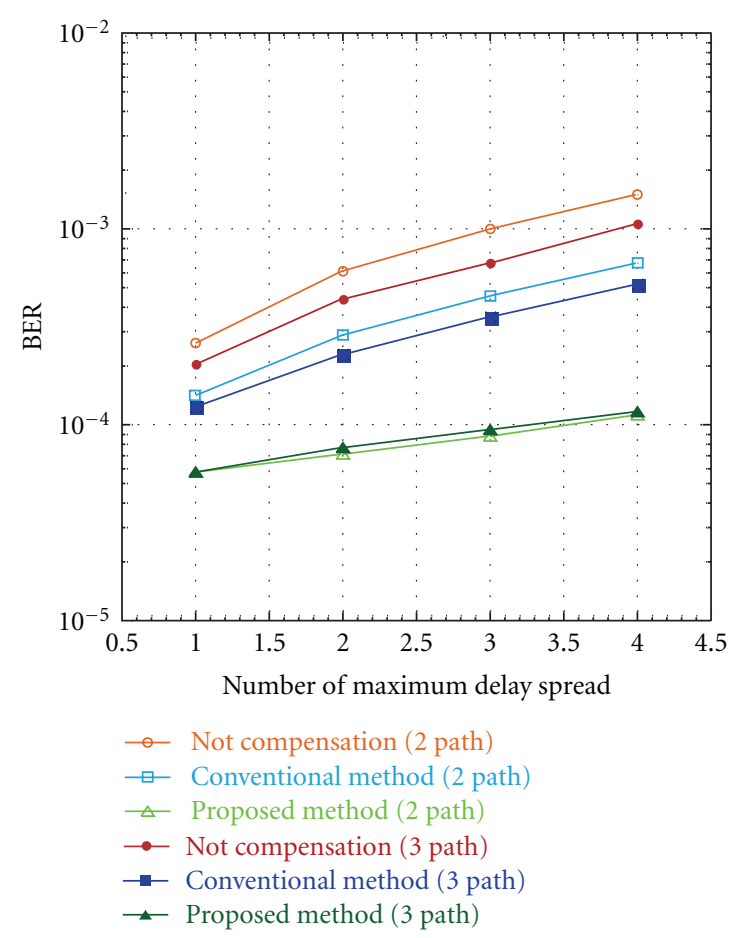

FIGURE 7: The BER versus the number of maximum delay spread for the conventional and proposed methods with $2 \times 2$ MIMO system at Doppler frequency of $10 \mathrm{~Hz}$.

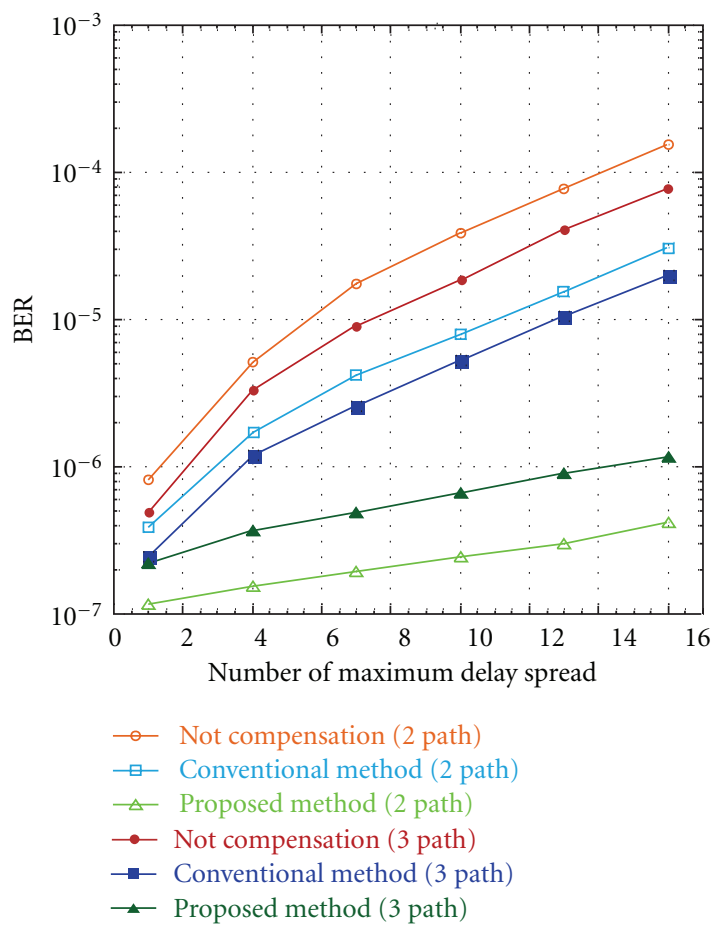

FIGURE 8: The BER versus the number of maximum delay spread for the conventional and proposed methods with $4 \times 4$ MIMO system at Doppler frequency of $10 \mathrm{~Hz}$. 


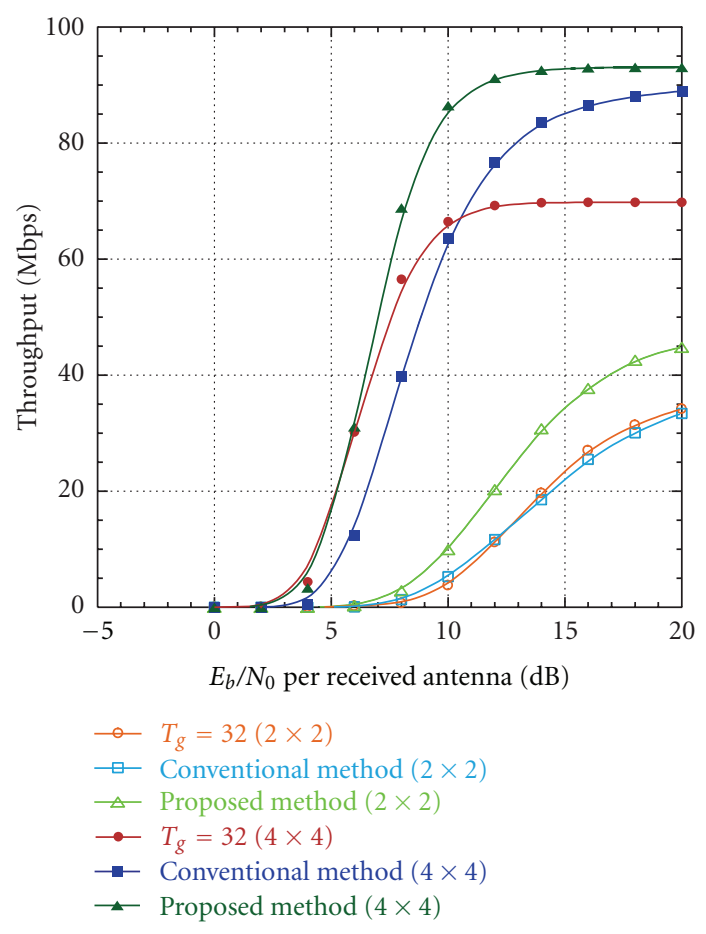

Figure 9: The throughput of the conventional and proposed methods for $2 \times 2$ and $4 \times 4$ MIMO systems at Doppler frequency of $10 \mathrm{~Hz}$.

of the proposed method with the 2-path model is increased about 36 times compared with 3-path model. This is because the channel impulse responses of the GI for the second time domain HTRCI-MIMO/OFDM pilot symbol are overlapped from the first pilot symbol in $4 \times 4$ MIMO system.

Figure 9 shows the throughput performance of the conventional and proposed methods for $2 \times 2$ and $4 \times 4$ MIMO systems at Doppler frequency of $10 \mathrm{~Hz}$. The number of maximum delay spread is 4 and 16 in $2 \times 2$ and $4 \times 4$ MIMO systems, respectively. The throughput $T_{t p}$ is given by

$$
T_{t p}=\frac{N_{d} \cdot N_{c} \cdot C \cdot R \cdot M}{\left(N_{p}+N_{d}\right) T} \cdot\left(1-P_{\mathrm{per}}\right),
$$

where $R$ is the coding rate and $P_{\text {per }}$ is the packet error rate (PER), respectively. The extension of the GI length is the simple method to prevent the problem of the maximum delay spread [13]. However, the throughput performance is degrade with this method. This is because the number of carriers $N_{c}$ decreases for (27) when the OFDM symbol length $T$ is the same as no ISI and ICI case. In this simulation, $N_{c}$ decreases from 64 to 48 , and the GI length $T_{g}$ increases from 16 to 32 . On the other hand, the proposed method can maintain the number of carriers $N_{c}$. From (27), the maximum throughout for no ISI and ICI case is about 48.8 and $93.1 \mathrm{Mbps}$ in $2 \times 2$ and $4 \times 4$ MIMO systems, respectively. Therefore, the proposed method shows the best throughput performance and can achieve the maximum throughput performance as the same no ISI and ICI case.

\section{Conclusion}

In this paper, we have focused on the large delay spread channel and proposed the ISI and ICI compensation methods for a HTRCI-MIMO/OFDM. In the proposed method, we have performed the time domain ISI compensation method with the replica signal based on the ICI compensation. From the simulation results, the proposed method has achieved the approximately same BER performance like the case with no ISI and ICI. Moreover, the proposed method can suppress the ISI and ICI due to the changing of the number of maximum delay spread. Finally, the proposed method has shown the best throughput performance and can achieve the same maximum throughput performance compared with no ISI and ICI case.

\section{References}

[1] "2010 White paper information and communications in Japan," http://www.soumu.go.jp/johotsusintokei/whitepaper/ ja/h22/pdf/index.html.

[2] H. Shoki and Y. Tanabe, "How fast will be required, how fast can be realized in future wireless communications ?" IEICE $B^{*}$ Pluse, no. 11, pp. 12-22, 2009.

[3] F. Adachi, H. Tomeba, and K. Takeda, "Introduction of frequency-domain signal processing to broadband single-carrier transmissions in a wireless channel," IEICE Transactions on Communications, vol. E92-B, no. 9, pp. 2789-2808, 2009.

[4] S. Abeta, "Toward LTE commercial launch and future plan for LTE enhancements (LTE-Advanced)," in 12th IEEE International Conference on Communication Systems (ICCS '10), pp. 146-150, November 2010.

[5] L. J. Cimini, "Analysis and simulation of a digital mobile channel using orthogonal frequency division multiplexing," IEEE Transactions on Communications, vol. 33, no. 7, pp. 665-675, 1985.

[6] J. A. C. Bingham, "Multicarrier modulation for data transmission: an idea whose time has come," IEEE Communications Magazine, vol. 28, no. 5, pp. 5-14, 1990.

[7] A. van Zelst, R. van Nee, and G. A. Awater, "Space division multiplexing (SDM) for OFDM systems," in 51st Vehicular Technology Conference (VTC '00), pp. 1070-1074, May 2000.

[8] I. Koffman and V. Roman, "Broadband wireless access solutions based on OFDM access in IEEE 802.16," IEEE Communications Magazine, vol. 40, no. 4, pp. 96-103, 2002.

[9] Y. Ogawa, T. Ohgane, and T. Nishimura, "MIMO technologies for ultra high-speed wireless transmission," IEICE B* Plus, no. 11, pp. 32-38, 2009.

[10] A. Ghosh, R. Ratasuk, B. Mondal, N. Mangalvedhe, and T. Thomas, "LTE-advanced: next-generation wireless broadband technology," IEEE Wireless Communications, vol. 17, no. 3, pp. 10-22, 2010.

[11] K. Zheng, L. Huang, G. Li, H. Cao, W. Wang, and M. Dohler, "Beyond 3G evolution," IEEE Vehicular Technology Magazine, vol. 3, no. 2, pp. 30-36, 2008.

[12] C. J. Ahn, "Achievable throughput enhancement based on modified carrier interferometry for MIMO/OFDM," Digital Signal Processing, vol. 20, no. 5, pp. 1447-1457, 2010.

[13] N. Gejoh and Y. Karasawa, "OFDM transmission characteristic in multipath environment where the delay spreading 
exceeds the guard interval analyzed based on the ETP model," IEICE Transactions on Communications, vol. J85-B, no. 11, pp. 1904-1912, 2002.

[14] S. Suyama, H. Suzuki, and K. Fukawa, "A MIMO-OFDM receiver employing the low-complexity turbo equalization in multipath environments with delay difference greater than the guard interval," IEICE Transactions on Communications, vol. E88-B, no. 1, pp. 39-46, 2005.

[15] D. Van Nguyen, "Interference cancellation for MIMO-OFDM systems in the case of insufficient guard interval length," in 1st International Conference on Communications and Electronics (ICCE '06), pp. 178-183, October 2006.

[16] Y. Ida, C. J. Ahn, T. Kamio, H. Fujisaka, and K. Haeiwa, "An interference cancellation scheme for TFI-OFDM in timevariant large delay spread channel," Radioengineering, vol. 18, no. 1, pp. 75-82, 2009. 

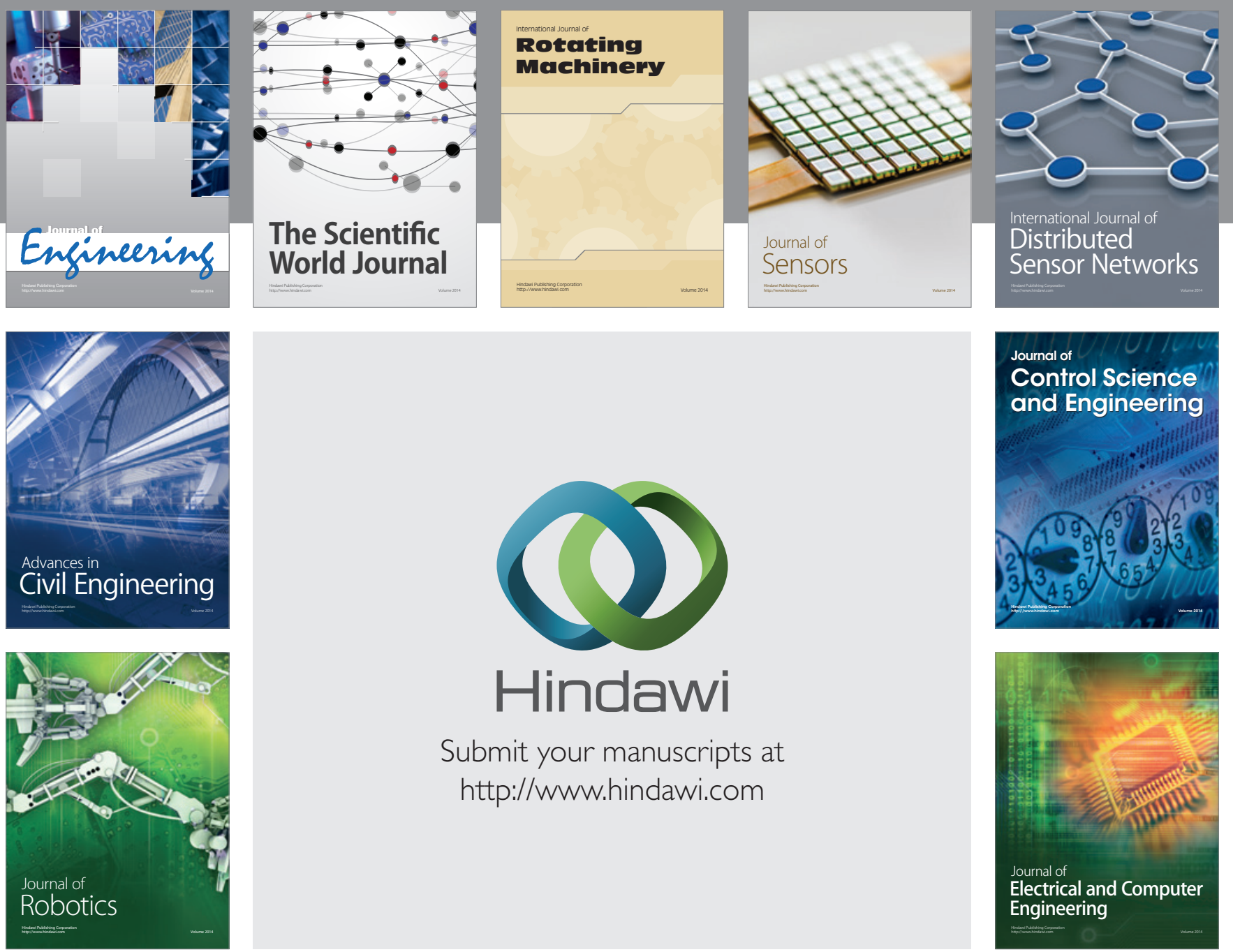

Submit your manuscripts at

http://www.hindawi.com
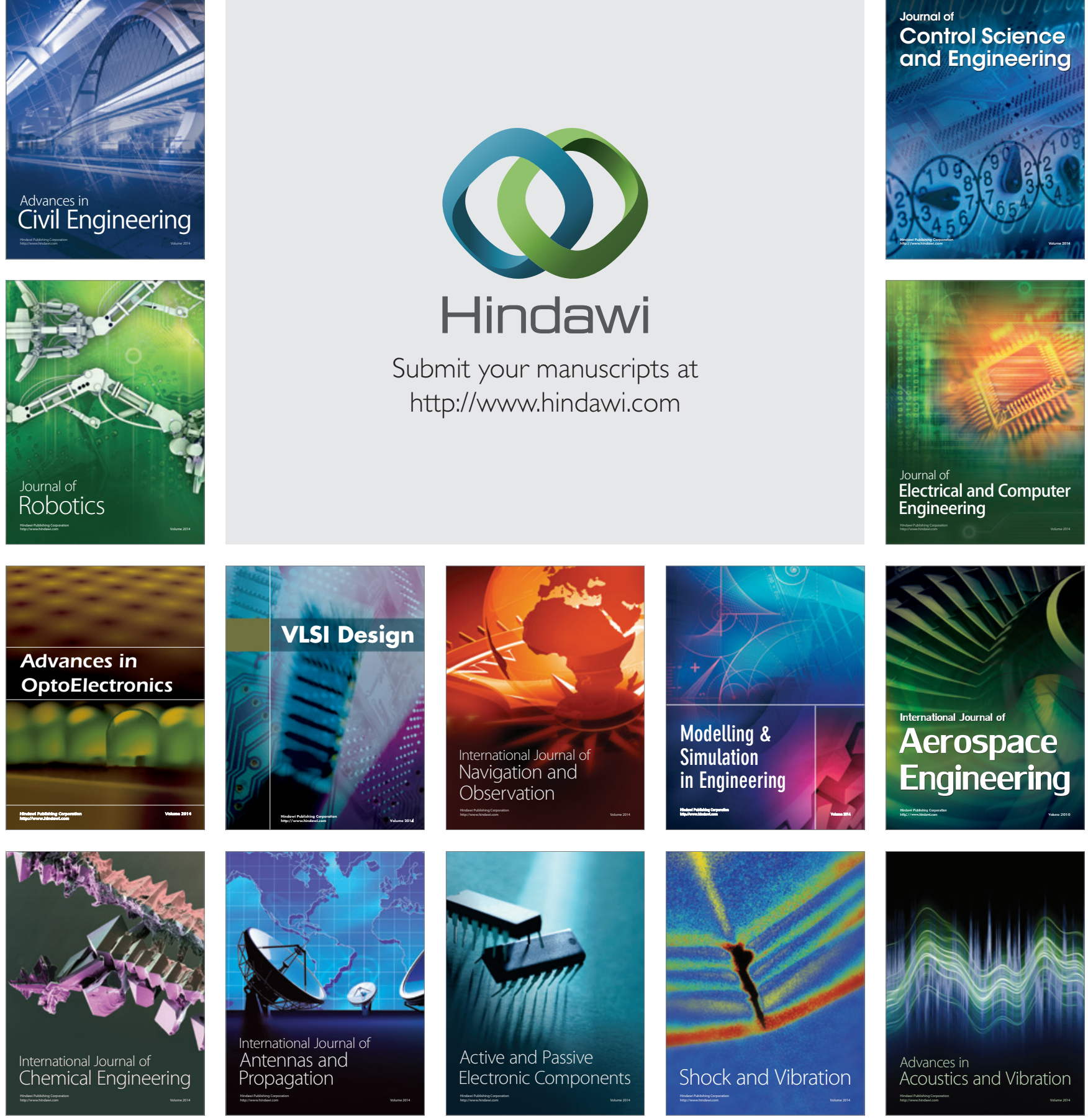